\title{
L'édification posthume de Ronsard en poète classique: de la consécration à la conservation
}

\section{François Rouget}

\section{(2) OpenEdition}

1 Journals

\section{Édition électronique}

URL : http://journals.openedition.org/studifrancesi/9774

DOI : 10.4000/studifrancesi.9774

ISSN : 2421-5856

Éditeur

Rosenberg \& Sellier

\section{Édition imprimée}

Date de publication : 1 août 2017

Pagination : 305-319

ISSN : 0039-2944

\section{Référence électronique}

François Rouget, «L'édification posthume de Ronsard en poète classique: de la consécration à la conservation », Studi Francesi [En ligne], 182 (LXI | II) | 2017, mis en ligne le 01 août 2018, consulté le 06 janvier 2021. URL : http://journals.openedition.org/studifrancesi/9774 ; DOI : https://doi.org/ 10.4000/studifrancesi.9774

\section{(c) (i) (9)}

Studi Francesi è distribuita con Licenza Creative Commons Attribuzione - Non commerciale - Non opere derivate 4.0 Internazionale. 


\author{
L'édification posthume de Ronsard \\ en poète classique: \\ de la consécration à la conservation
}

\begin{abstract}
Pierre de Ronsard died in 1585 leaving to posterity the massive volume in-folio of his CEuvres (Paris, G. Buon, 1584). In the subsequent nine editions published between 1587 and 1630, his editors augmented his poetry with commentaries and typographical components that enhanced his figure as a "classical" author. This article presents the various strategies explored by Ronsard's editors to convey his image as the «Prince of poets». While preserving his poems in a faithful way that Ronsard defined before his death, his admirers seeked to disseminate his poetry through literary and musical anthologies as well as visual imagery.
\end{abstract}

De son vivant, Pierre de Ronsard (1524-1585) avait pris en main l'édition et la diffusion de ses Euvres qu'il réunit à diverses étapes de sa carrière pour intégrer au massif de sa poésie collective des recueils publiés séparément. C'est ainsi qu'il fit paraître les éditions de ses Euvres toujours augmentées entre 1560 et 1584, date de la dernière publiée de son vivant. Soucieux de livrer à la postérité une œuvre monumentale, Ronsard choisit alors le format de l'in-folio, redistribue ses pièces dans des divisions renouvelées, et fait appel aux graveurs pour présenter de nouvelles illustrations qui promeuvent sa persona.

Cette stratégie éditoriale est bien connue de la critique universitaire ${ }^{1}$. Ce qui l'est moins est la diffusion posthume de l'œuvre qui se poursuivit à un rythme soutenu jusqu'en 1630, cela en dépit du renouvellement de la poésie et du goût littéraire qui apparut dans le premier quart du XVII siècle. Devenu une figure classique de la poésie de la Renaissance grâce à son talent littéraire et à l'action conjuguée du poète, de ses éditeurs et de ses lecteurs, Ronsard avait confié à ses légataires universels le soin de perpétuer la survie de ses Euvres. C'est ce qu'ils firent en multipliant les rééditions de celles-ci (huit entre 1587 et 1630) et en adaptant leur impression et leur diffusion en fonction des conditions nouvelles. S'attachant à livrer l'image d'un auteur classique à l'œuvre éminemment moderne, actuelle, les éditeurs de Ronsard ont su recourir à tous les moyens de la publication éditoriale pour rehausser la gloire de l'homme et la mémoire de son œuvre.

Dans la présente étude, nous voudrions examiner les stratégies éditoriales mises en œuvre par les éditeurs de Ronsard pour perpétuer le succès de sa poésie après sa mort de 1587 à 1630 . Il s'agira d'étudier les caractéristiques de la «mise en livre»² de

(1) Sur cette question, voir M. Simonin, Ronsard et la poétique des "CEuvres", in Ronsard en son IV centenaire: I. Ronsard hier et aujourd'bui, éd. Y. Bellenger, J. Céard, D. Ménager et M. Simonin, Genève, Droz, 1988, pp. 47-59 (repris dans L'Encre et la lumière. Quarante-sept articles (1976-2000), Genève, Droz, 2004, pp. 237-251), et F. ROUGET, Ronsard et le Livre. Étude de critique génétique et d'bistoire littéraire, 2 t., Genève, Droz, 2010 et 2012.

(2) Sur cette notion, voir R. Chartier, Du livre au lire, in Pratiques de la lecture, R. Chartier (dir.), ParisMarseille, Rivages, 1985, p. 80. 
l'œuvre (choix des formats, recours à l'iconographie, ajout de commentaires), des agents de sa production (libraires, imprimeurs, éditeurs scientifiques, illustrateurs) et des facteurs et modalités de sa diffusion (calendrier des éditions, recherche des inédits, diffusion exogène dans les anthologies poétiques, didactiques ou musicales). Si le souvenir de la personne et de la poésie de Ronsard demeurait si vif dans la mémoire des lecteurs du XVII ${ }^{e}$ siècle qui en collectionnaient les ouvrages, c'est que les éditeurs de sa poésie surent en perpétuer la dimension classique, celle d'un poète "à l'antique", et en accroître la diffusion auprès d'un public élargi (humanistes, courtisans, bourgeois, collégiens) au moyen des ressources nouvelles offertes par l'imprimerie.

\section{La «classicisation» de Pierre de Ronsard}

Épris de gloire littéraire dès son entrée en scène vers 1549, le jeune Ronsard n'avait pas attendu pour acquérir la dimension classique qu'on lui connait. À vrai dire, c'est lui qui prit en main son destin en s'entourant de collaborateurs qui furent conviés à rehausser son image et à conforter sa réputation. Soucieux de la diffusion de ses textes autant que de son image publique, il s'attacha dès 1550 à faire entrer son œuvre parmi celles des classiques. Il procéda de plusieurs façons, à la fois par le choix des genres poétiques, des supports matériels de diffusion et des associés qui assurèrent la réception de l'œuvre.

Les premiers poèmes de Ronsard s'inscrivirent dans la poésie officielle de cour pour célébrer l'avènement d'Henri II. L'Avantentrée du roi treschrestien à Paris (1549), L'Hymne de France (1549), furent des plaquettes qui préparèrent la parution d'un recueil plus ambitieux et varié, Les Quatre premiers livres des Odes (1550). Ronsard entendait apparaître comme un poète classique de langue moderne ayant adapté en français les grands lyriques de l'Antiquité (Pindare et Horace). Les autres genres littéraires qu'il choisit par la suite - sonnets, hymnes, épopée - renforcèrent cette image d'un écrivain poursuivant la tradition lyrique. Dans le recueil des Odes de 1550, Ronsard est vanté comme un nouveau Terpandre par une cohorte d'admirateurs qui lui consacrèrent de nombreux vers latins et grecs. Ronsard souhaitait clairement s'écarter de ses rivaux pour faire «stile apart, sens apart, euvre apart»" et situer son écriture dans la veine de la poésie humaniste. Les autres compositions qu'il fit paraître ensuite multiplièrent les allégeances au canon poétique. En 1552, ses Amours sont placées sous le signe de Pétrarque, le grand modèle de la lyrique amoureuse devenu un classique dès l'apparition du livre. Ronsard va même jusqu'à emprunter aux éditions italiennes du Canzoniere l'exemple des représentations du poète-amant et de sa dame. S'il n'est pas le premier Français à s'inspirer de cette iconographie, il semble toutefois vouloir se rapprocher de Pétrarque en s'inspirant fidèlement de ses vers et de l'image que l'Italien se dessine dans la conscience littéraire4. En 1552, Ronsard non seulement fait suivre ses Amours d'un Cinquieme livre des Odes (pièces majestueuses et graves qui rehaussent la légèreté faussement apparente des sonnets amoureux) mais aussi leur adjoint un Supplément musical qui permet de chanter les sonnets et d'élargir le public potentiel des lecteurs. La préparation soignée de cette édition

(3) Avis «Au lecteur» en tête des Odes (1550), ROnSARD, Euvres complètes, sous la direction de P. Laumonier, R. Lebègue et I. Silver, Paris, STFM, t. I, 1973, p. 45. Toutes nos références renvoient à cette édition.

(4) Comme l'a bien montré D. MAIRA, dans sa thèse Typosine, la dixième muse. Formes éditoriales des canzonieri français (1544-1560), Genève, Droz, 2007. 
suggère un effort concerté de Ronsard et de ses collaborateurs (M. de La Porte, le libraire, et M.A. Muret, C. Janequin, P. Certon et C. Goudimel, les musiciens) pour produire un recueil de poésie unanimement reconnu comme un livre important. Nul doute que c'est Ronsard - peut-être de connivence avec Muret - qui prit les devants et commanda la participation des musiciens, alors qu'à cette époque l'initiative était laissée d'habitude à ceux-ci pour sélectionner les pièces qui figureraient dans leurs anthologies musicales 5 .

Fort de ce premier succès, Ronsard prolonge l'expérience "humaniste" de la diffusion poétique. Un an après la parution de l'édition princeps des Amours, il publie une seconde édition accompagnée pour l'occasion d'un Commentaire rédigé par M.A. Muret. À l'instar des éditions commentées de Pétrarque et des éditions des classiques gréco-latins, la poésie ronsardienne se voyait encadrée par un appareil critique et herméneutique justifié par la nature sublime de l'inspiration et le caractère abscons du style poétique. Par cette adjonction, Ronsard entendait sûrement éclairer les passages qui avaient déconcerté son lectorat; il cherchait aussi à hisser le recueil des Amours au rang de texte classique, consacré déjà par la tradition philologique ${ }^{6}$.

Cette démarche conjointe du poète et de son interprète, Ronsard la poursuivit alors qu'il préparait l'édition collective de ses Euvres (1560). Fait notable, pour la première fois, un poète réunissait et organisait de son vivant tout ce qu'il avait déjà publié et il ajoutait de nouvelles compositions. Même Clément Marot, père de la poésie française depuis le règne de François $I^{\text {er }}$, n'était parvenu à réaliser ce qui pouvait apparaître comme un péché d'orgueil. Seuls les grands auteurs classiques pouvaient espérer bénéficier du privilège de connaître leurs opera réunies et publiées après leur mort. Tandis qu'il avait atteint sa trente-sixième année, donc à l'âge de la maturité, Ronsard s'empressait de livrer en quatre tomes in-12 la première collective de ses Euvres, qui allait marquer une longue série. Cette édition était sans doute dictée par la crainte de disparaître soudainement (car Ronsard était souffrant) et motivée par la mort récente de Joachim Du Bellay, l'ami et le confrère le plus proche ${ }^{7}$. Mais n'avaitelle pas aussi pour objectif de regrouper, d'élaguer et d'organiser des poésies diverses auxquelles il s'agissait alors de donner un sens et une forme cohérente?

Ronsard profita donc d'un nouveau "privilège” royal accordé par François II pour livrer une édition-bilan qui apparaît bien comme un aboutissement et un nouveau départ ${ }^{8}$. Il charge Rémy Belleau de commenter le Second livre des Amours, afin de compléter le travail réalisé par Muret sur le Premier livre de cette section'. Il réimprime des éloges liminaires, en accueille de nouveaux, mais il est présenté dans un portrait qui le dépeint en gentilhomme du XVI ${ }^{\mathrm{e}}$ siècle dont la tête est ceinte d'une cou-

(5) Le Supplément musical est reproduit dans l'éd. citée, t. IV, 1982, pp. 189-250; voir aussi l'édition procurée par Chr. DE Buzon et P. MarTin, Les Amours, leurs Commentaires (1553), Paris, Didier, 1999 , pp. 294-355.

(6) Cet aspect a été relevé par la critique; voir notamment, dans l'éd. Chr. de Buzon et P. Martin des Amours (1553), l'étude de J. CÉARD, Muret, commentateur des "Amours" de Ronsard, pp. 359-379, et surtout les Commentaires au Premier livre des Amours de Ronsard publiés par J. Chomarat, M.-M. Fragonard et G. Mathieu-Castellani, Genève, Droz, 1985.

(7) M. Dassonville a le premier étudié les circonstances de publication et l'organisation intime de cette édition; voir Ronsard. Étude historique et littéraire. Tome IV: Grandeurs et servitudes, Genève, Droz, 1985, chap. III, pp. 77-108.

(8) Sur cette question, voir F. Rouget, Ronsard et le Livre cit., t. II, pp. 47-64, et M. ClÉmEnT, Les poètes et leurs libraires au prisme du privilège d'auteur au XVI siècle: la proto-propriété littéraire, in Les Poètes français de la Renaissance et leurs "libraires", éd. D. Bjaï et F. Rouget, Genève, Droz, 2015, pp. 30-39.

(9) On peut le lire dans Le Commentaire au Second livre des Amours de Ronsard par R. Belleau, éd. M.M. Fontaine et F. Lecercle, Genève, Droz, 1986. 
ronne de laurier, symbole de sa consécration. Par là, Ronsard apparaît à la fois comme un auteur vivant et dont l'œuvre a déjà rejoint le canon classique de la tradition.

Cette image, il la cultiva sans cesse dans la production de ses recueils successifs. À chaque fois, il s'agissait pour lui de publier de nouveaux ouvrages (Les Hymnes en 1555-1556; les Discours en 1562-1563, etc.) et d'intervenir sur la scène littéraire pour la dominer en poète de la modernité; à intervalles réguliers, cependant, il rassemble aux textes antérieurs les nouvelles productions pour procurer des éditions collectives mises à jour (en 1567, 1571, 1572-1573, 1578) par lesquelles il prépare sa réception posthume. Peu avant sa mort, il ambitionne même de rassembler ses textes en une édition in-folio, dont le format rappelle celui des Opera des plus grands auteurs de l'Antiquité. C'est par elle que Ronsard entend se livrer à la postérité, même s'il la révise peu après, alors qu'elle vient de paraître. En tout cas, ce volume de 1584 imprimé sur beau papier suggère la canonisation du vivant de Ronsard dont l'effigie - par le portrait révélé dans les Amours (1552), puis repris en tête de La Franciade (1572) en face de celui de Charles IX - est placé au seuil de l'œuvre, juste avant le début de la section des Amours (fig. 1).

Salué durant sa vie par ses confrères dans leurs propres œuvres poétiques, traduit en latin par ses admirateurs (J. Dorat, F. de Thoor, etc.), cité en modèle par les grammairiens et les auteurs d'arts poétiques et de rhétorique (P. de La Ramée, A. Fouquelin), élu par les musiciens de son temps et les compilateurs d'anthologies poétiques $^{10}$, Ronsard a laissé tout au long de sa carrière les traces d'une classicisation de son œuvre de poète. Rénovateur de la tradition, il s'est efforcé de rejoindre celle-ci en se posant comme l'exemple même du grand écrivain en langue vernaculaire, témoin de son temps et des exploits des grands hommes ${ }^{11}$. Une bonne partie de son existence aura été consacrée à l'élaboration d'une langue française permettant d'accueillir les grands genres du passé et de les adapter dans des formes modernes car renouvelées.

S’il n'a laissé à quiconque le soin de publier ses œuvres jusqu'à sa mort survenue au début de l'hiver 1586, il lui aura pourtant fallu compter sur le service fidèle de ses plus proches amis - désignés par lui comme les légataires universels de son œuvre - pour perpétuer sa mémoire et poursuivre la diffusion de ses Euvres à partir de 1587.

\section{La perpétuation posthume des "Euvres": consécration et conservation}

Malgré tous les efforts qu'il avait déployés au cours de sa carrière pour la rendre vivante, Ronsard avait bien conscience de la fragilité de son œuvre poétique. En dépit de ses déclarations fracassantes aux Grands, dispersées dans ses vers de cour dès 1550, qui s'efforcent de les convaincre de la supériorité de la poésie sur l'architecture, le livre de papier ne résiste pas mieux que le palais de pierre à l'érosion du temps. L'eau, le feu, les pillages des guerres sont les menaces de la poésie que seule la mémoire des hommes peut sauver de l'oubli.

Le poète vendômois a alors mis tout en œuvre pour assurer la pérennité de ses textes en laissant à d'autres le soin de les rééditer à intervalles réguliers après sa dispa-

(10) Sur ces divers aspects de la diffusion des textes de Ronsard hors de ses livres, voir F. RougET, Ronsard et le Livre cit., t. II, p. 245 ss., p. 394 ss., p. 411 ss., p. 423 ss., et p. 457 ss.

(11) Sur cette alliance intrinsèque de l'écrivain et des Grands, voir F. Rouget, Ronsard et le grand bomme, in «Travaux de Littérature» XVIII, 2005, pp. 87-103. 
rition. Peu avant son décès à la fin de décembre 1585, il choisit Jean Galland et Claude Binet comme légataires universels de ses Euvres $^{12}$. Ceux-ci deviennent les détenteurs du privilège général acquis par Ronsard et ils s'assureront de révéler les textes inédits et de faire réimprimer les Euvres en fonction des notes laissées par le poète défunt. Autant dire que dès 1585 , tout est mis en place pour garantir la perpétuation de la gloire littéraire de cet homme, et cela est orchestré en partie par Ronsard lui-même. Jean Galland et ses successeurs veilleront à faire renouveler le privilège éditorial chaque fois qu'il sera échu, notamment en 1587,1597 , etc. ${ }^{13}$. Cette continuité éditoriale est assurée par la collaboration des éditeurs scientifiques Jean puis Philippe Galland (à partir de 1612), et de la famille de libraires Buon (Gabriel puis Nicolas) et de leurs associés (B. Macé) et successeurs (S. Thibout, R. Baraigne).

Ce qui frappe est l'énergie avec laquelle Claude Binet et Jean Galland organisent la célébration de la mort de Ronsard. Tous les moyens sont mis en œuvre pour organiser des funérailles somptueuses, tous les admirateurs et contemporains du poète sont conviés à faire résonner partout dans le royaume la nouvelle tragique, qui était pourtant attendue - parfois avec un plaisir non dissimulé par ses adversaires protestants. Il n'est donc guère étonnant que les lettrés les plus réputés (J. Davy Du Perron, G. Critton, J. Viellard, etc.) comme les moins connus se soient mobilisés pour publier tombeaux en prose et en vers, et en plusieurs langues, en divers lieux ${ }^{14}$. Étant donné la place qu'il occupait à la Cour et sur la scène littéraire, la disparition de Ronsard fut perçue en effet comme un événement de première importance et qui justifiait des funérailles nationales. Mais il y avait plus pour perpétuer le souvenir d'un poète qu'on souhaitait presque faire parler au-delà du tombeau: Claude Binet, qui avait partagé les derniers instants du poète, réunit ses Derniers vers, quelques sonnets et courtes pièces composés sur son lit de mort, pour les imprimer et les distribuer aux admirateurs qui assistèrent aux funérailles parisiennes. Parus simultanément avec l'hommage public, ces vers venaient démentir poétiquement et symboliquement la disparition de Ronsard et l'arrêt de l'œuvre.

Cette image d'un poète mort-vivant allait être cultivée par Binet et Galland qui révélèrent au cours des années suivantes certains textes ronsardiens inédits justifiant la réédition «augmentée» des Euvres collectives, et qui développèrent une véritable hagiographie ronsardienne. La première étape fut la publication dès 1586 du Discours de la vie de P. de Ronsard par Claude Binet. Construite sur quelques confidences personnelles de l'homme et nourrie de nombreux souvenirs empruntés à ses poèmes, cette biographie insistait sur le caractère exceptionnel de Ronsard. Constamment corrigé et réédité (notamment en 1587 et 1597), le Discours de Binet rejoignit la première collective posthume des CEuvres pour ne plus les quitter. À l'image des grands textes de la tradition littéraire, l'œuvre ronsardienne s'accompagnait d'une vita dont la fonction double consistait autant à présenter la vie d'un homme auprès du nouveau public né après 1585 , qu'à expliquer les valeurs d'une esthétique qui pouvait parâtre périmée aux yeux du lectorat de Malherbe.

Après les célébrations funéraires qui avaient culminé au cours de l'hiver 1586, et peu après l'établissement de la biographie officielle ou autorisée de Ronsard, une nouvelle édition des Euvres vit le jour fin décembre de 1586 (bien que la date de 1587 figurât sur les tomes), alors que tous les exemplaires de la précédente n'étaient

(12) Sur les relations privilégiées entre Ronsard et ses deux admirateurs, voir C. BINET, Discours de la vie de P. de Ronsard, éd. P. Laumonier, Paris, 1909 (et Genève, Slatkine Reprints, 1969), p. 29 ss.

(13) Sur l'état de cette question, voir F. Rouget, Ronsard et le Livre cit, t. II, pp. 59-64.

(14) M. Simonin dans l'Introduction de son édition de l'Oraison funebre sur la mort de Monsieur de Ronsard de J. Davy Du Perron (1586) (Genève, Droz, 1985) dresse la liste de ces hommages rendus à Ronsard. 
sans doute pas tous écoulés ${ }^{15}$. Cette précipitation s'explique par la volonté des légataires universels de contrôler la diffusion de l'œuvre. À l'occasion de la parution de cette première collective posthume, vingt-sept inédits furent révélés et de nombreux amendements furent apportés aux textes d'après, semble-t-il, les indications laissées par Ronsard avant sa mort. Les dix tomes de cette édition in-12 réorganisaient quelque peu la distribution des poèmes et accueillaient le Discours de la Vie de Ronsard par Binet vantant sa connivence avec le poète et l'autorité morale qu'il exerçait sur ses Euvres ${ }^{16}$. En d'autres termes, Galland et Binet entendaient garantir l'intégrité de l'œuvre ronsardienne mais aussi tirer un certain prestige de leur statut de gardiens de sa mémoire. La disparition du poète eût pu reléguer l'œuvre dans le domaine public et laisser à tout libraire le loisir de la réimprimer à sa guise. Malgré le privilège dont jouissaient Galland et Binet, l'œuvre fut pourtant pillée en 1592 par le libraire lyonnais Thomas Soubron qui réimprima la totalité des Euvres avec les pièces retranchées du vivant de Ronsard. L'instigateur de cette édition, Antoine Du Verdier, comme le suppose M. Simonin ${ }^{17}$, profitait du flou encadrant la législation sur l'impression des livres pendant la Ligue pour s'emparer de l'œuvre ronsardienne et rivaliser avec les éditions légitimes parisiennes en procurant la réunion la plus complète possible des textes composés par Ronsard. La réplique ne se fit guère attendre; dans la réédition des Euvres en 1597, Buon condamna l'indélicatesse de son confrère lyonnais et surenchérit en livrant quelques inédits et en les agrémentant de nouveaux commentaires.

Dès lors, Buon et ses associés vont multiplier les rééditions augmentées d'inédits et de commentaires pour déjouer toute tentative de plagiat fomentée par leurs rivaux. En 1604, la troisième collective autorisée des Euvres vit le jour qui ne contenait pourtant qu'une pièce inédite, mais des commentaires abondants rédigés notamment par N. Richelet pour les Odes et J. Besly pour les Hymnes ${ }^{18}$. Cinq ans plus tard, une double opération promotionnelle fut orchestrée par les éditeurs: une édition in-folio parut conjointement à une édition de petit format (in-12). Malgré quelques divergences, les deux versions sont similaires. Ce qui les différencie cependant est leurs formats qui les destinent à deux types de lecteurs, l'un disposant de moyens financiers supérieurs à l'autre. Ce coup double permettait sans doute de produire une édition de luxe, réservée à une élite, à côté d'une édition plus commode et bon marché. Mais le plus notable est la volonté des éditeurs de procurer tous les textes connus de Ronsard en constituant une dernière partie des Euvres intitulée Recueil des sonnets, odes, bymnes, elegies, gragments et autres pieces retranchées aux editions precedentes des ceuvres [...]. Avec quelques autres non imprimées cy devant, partie dans laquelle on retrouvait les poèmes que $\mathrm{Du}$ Verdier avait rétablis dès 1592. L'intention est ici clairement d'offrir une moisson ample et renouvelée comme pour livrer de l'œuvre un visage rafraîchi. Les rééditions suivantes s'inscrivent dans la même démarche puisqu'en 1617 le Recueil est grossi d'une trentaine de pièces en vers et en prose.

À partir de cette date, l'entreprise éditoriale devient archéologique et philologique. Outre les pièces ajoutées, de nouveaux commentaires entrent dans les CEuvres, notamment ceux de Richelet sur les Hymnes. Pour accueillir tous ces textes qui viennent se greffer sur le massif des Euvres, et que Ronsard n'avait paradoxalement

(15) On peut lire cette édition importante dans l'édition moderne procurée par I. SILVER, Euvres complètes de Ronsard, Chicago, The University of Chicago Press, et Paris, Didier, 1966-1970, 8 t.

(16) Voir F. Rouget, Ronsard et le Livre cit., t. II, p. 551 et ss.

(17) Les contrefaçons lyonnaises de Montaigne et Ronsard au temps de la Ligue, in Les Presses grises, Paris, Aux Amateurs de Livres, 1988, pp. 139-159 (étude reprise dans L'Encre et la lumière, op. cit., pp. 783-802).

(18) Sur la genèse des commentaires posthumes, voir F. Rouget, Ronsard et le Livre cit., t. II, p. 217 ss. 
pas souhaités, les éditeurs décident d'un format monumental. En 1623, c'est donc non pas un mais deux volumes in-folio qu'il faut pour imprimer la sixième collective des Euvres. Les lettrés de l'époque sont mobilisés, Nicolas Richelet mais aussi Pierre de Marcassus et Claude Garnier, pour produire de nouveaux commentaires censés éclairer et rehausser le sens de la poésie ronsardienne ${ }^{19}$. L'apparat critique est si abondant qu'il envahit parfois l'espace de la page au point de reléguer le texte poétique au second plan. Conservé, redécouvert et réimprimé, commenté à l'envi, le texte ronsardien étouffe et devient prétexte à l'intervention de commentateurs dont l'image s'affiche à côté de celle de Ronsard. À vouloir pérenniser à tout prix la poésie et la légende ronsardiennes, ses hagiographes ont fini par statufier le poète en commandeur. La classicisation du poète et de son œuvre, qui relevait sans doute d'une intention louable, s'effectuait peut-être quelque peu aussi aux dépens de la «poétique des Euvres» voulue par Ronsard. Sans cesse augmentée, sa poésie se voyait défigurée; peuplée de voix étrangères qui assourdissaient celle de Ronsard, l'œuvre perdait son attrait original et sa physionomie primitive, et avec elle un peu de sa fraîcheur.

C'est peut-être aussi que Ronsard était lu à présent avec les yeux de lecteur d'une nouvelle époque, jugeant le lyrisme à l'aune de la langue de Malherbe. On sait comment celui-ci considérait les Premieres Euvres de Philippe Desportes; il serait tout aussi éclairant de découvrir un jour l'exemplaire des Euvres ronsardiennes annotées par Malherbe. On le verrait peut-être reprendre son modèle et démystifier la conception sublime et quasi oraculaire de sa poésie. Car Ronsard est présenté au public du premier quart du XVII ${ }^{\mathrm{e}}$ siècle sous les traits d'un poète lyrique et épique, associant les vertus d'Homère et de Virgile ${ }^{20}$. C'est ce que révèle avec éloquence le frontispice gravé par Léonard Gaultier pour orner le volume in-folio des Euvres en 1609 (fig. 2). Placé en position supérieure, il occupe une place privilégiée pour inviter le lecteur contemporain à franchir le seuil du livre; situé sur un piédestal, il se présente comme le juge de toute future production en langue française. L'iconographie - qui constitue un facteur de la classicisation de l'écrivain - ne cesse de crôitre à la fin du $\mathrm{XVI}^{\mathrm{e}}$ siècle, notamment grâce à la généralisation de la gravure sur cuivre. On a vu que Ronsard avait pris les devants en associant son effigie à celle de ses protecteurs ou de ses égéries dès 1552. Après sa mort, sans doute pour donner un visage à un écrivain oublié ou méconnu, les éditeurs de ses Euvres multiplient les représentations de Ronsard en poète sacralisé par la tradition. Le frontispice de Gaultier est repris en totalité pour la huitième collective de 1623 et sous forme réduite pour la dernière de 1629-1630. Mais c'est aussi cette image qu'il reprend pour illustrer la page de titre de l'anthologie des Marguerites poetiques (Lyon, B. Ancelin, 1613; fig. 3) où Ronsard figure aux côtés d'un autre auteur à succès de l'époque, Guillaume Salluste Du Bartas. Ronsard avait rejoint l'Histoire depuis 1600 au moins, comme le suggère la suite de vignettes de la Chronologie collée où le buste de Ronsard apparaît au début de la septième ligne ${ }^{21}$. Représenté en tableaux, dessins, en médailles, Ronsard se devait d'être reconnu comme personnage public de son vivant, puis historique après

(19) Sur le rôle de ces exégètes, voir F. ROUGET, La réception de Ronsard d'après les commentaires aux éditions posthumes de ses "Euvres", in La Poésie de la Pléiade. Héritage, influences, transmission (Mélanges offerts au professeur Isamu Takata par ses collègues et ses amis), Y. Bellenger, J. Céard et M.-Cl. ThomineBichard (dir.), Paris, Éditions Classiques Garnier, 2009, pp. 311-326.

(20) Sur l'iconographie ronsardienne, voir l'étude de M.-M. FragOnARD, Ronsard en poète: portrait d'auteur, produit du texte, in Figures du poète. Pierre de Ronsard, M.-D. Legrand et J.-M. Maulpoix (dir.), Actes du colloque de l'ÉNS de Fontenay/Saint-Cloud et de l'Université de Paris X-Nanterre, avril 1999, Presses de l'Université de Paris X, «Littérales», 2000, pp. 15-41; voir aussi F. Rouget, Ronsard et le Livre cit., t. II, p. 604 ss.

(21) La vignette est reproduite par M.-M. Fragonard, art. cité [h. t.], et par F. Rouget, ibid., p. 617. 
sa mort. Icône de la poésie européenne, comme aime à le rappeler Jacob Boissard (Icones, Francfort, M. Becker, 1597), il est resté l'une des sources d'inspiration de la poésie du siècle suivant et a offert aux peintres la matière pour glorifier la monarchie française. En dépit de l'évolution du goût qu'on observe sous le règne d'Henri IV, les compositions picturales inspirées de La Franciade ronsardienne, que Toussaint Du Bray destinait aux galeries et appartements du Château-Neuf de Saint-Germain-enLaye, en constituent une preuve ${ }^{22}$. Chef de file de la poésie française du Xvi siècle, sans pour autant faire oublier l'autorité de Clément Marot et la concurrence de ses contemporains (Desportes et Du Bartas), Ronsard reste le maître à penser et à composer des vers. Après sa disparition, ses vers sont réédités sans cesse soit en totalité jusqu'en 1630, soit choisis pour rejoindre des anthologies poétiques qui fleurissent vers 1600-1620 - dans des recueils de poésie profane (Sejour des Muses, Tresor des chansons amoureuses, La Muse folastre), voire satirique (Le Cabinet satyrique), autant que des volumes de poésie sacrée (Muse chrestienne) ${ }^{23}$. La voix de Ronsard résonne partout et hors de ses CEuvres: sa poésie sert encore à l'inspiration des musiciens qui la joignent à celles de Desportes dans leurs anthologies imprimées ${ }^{24}$, et les rhéteurs et poéticiens la commentent ou l'imitent abondamment (P. de Deimier, Académie de l'Art poetique, Paris, 1610; J. Vauquelin de La Fresnaye, Art poetique, Caen, 1605). La poésie ronsardienne sert d'étalon pour mesurer toute évolution future et à partir de laquelle chacun est amené à définir sa voix propre.

Enfin, si ses Euvres ne seront plus rééditées en 1630 et ce jusqu'au XIX ${ }^{\mathrm{e}}$ siècle, tombant soudain dans l'oubli avec presque toute la littérature du XVI ${ }^{\mathrm{e}}$ siècle ${ }^{25}$, les exemplaires de ses éditions anciennes seront convoités des collectionneurs et figureront longtemps dans la bibliothèque des lettrés tout au long des XVII ${ }^{\mathrm{e}}$ et XVIII ${ }^{\mathrm{e}}$ siècles. C'est là un aspect méconnu de la réception de Ronsard et qui mérite un examen approfondi ${ }^{26}$. Car à côté des textes fondateurs de la littérature en langue française, édités depuis le début du XVI ${ }^{\mathrm{e}}$ siècle - les récits de chevalerie, le Roman de la Rose, Alain Chartier, les farces et moralités, les Rhétoriqueurs, Clément Marot -, figurent les nouveaux auteurs "classiques" tels Rabelais, Ronsard et Montaigne. Si l'œuvre poétique du Vendômois n'est plus réimprimée après 1630 , c'est sans doute que de nouveaux genres et auteurs venaient l'éclipser (le théâtre surtout avec Molière, Corneille et Racine), que le goût de la littérature classique lui substituait de nouvelles règles linguistiques plus actuelles, et aussi que l'abondance des exemplaires produits au cours des rééditions des Euvres (six de 1604 à 1630) suffisait pour répondre à la demande. En comparaison avec les six éditions collectives parues du vivant de Ron-

(22) Sur cette question, on consultera la mise au point de D. CORDELLIER, Dubreuil, peintre de la "Franciade" de Ronsard au Château-Neuf de Saint-Germain-en-Laye, in «Revue du Louvre et des Musées de France» 5/6, 1985, pp. 357-378.

(23) Ces florilèges ont fort bien été répertoriés et analysés par F. LACHÈvRE dans sa Bibliographie des recueils collectifs de poésies au XVI $I^{e}$ siècle (du Jardin de Plaisance, 1502, aux Recueils de Toussaint du Bray, 1609), Paris, Champion, 1922, et sa recension des Recueils collectifs de poésies libres et satiriques publiés depuis 1600 jusqu'à la mort de Théophile (1626), Paris, Champion, 1914.

(24) Comme le montre bien la Bibliographie des poésies de P. de Ronsard mises en musique au XVI siècle, préparée par G. Thibault, G. et L. Perceau, Paris, Droz, 1941, p. 73 ss. (pour les recueils musicaux publiés après la mort du poète).

(25) Sur cette histoire, voir la thèse de Cl. Faisant, Mort et résurrection de la Pléiade, Paris, H. Champion, 1998.

(26) Voir F. Rouget, Ronsard et le Livre cit., t. II, chap. VIII, pp. 623-660, et D. MAIRA, Des bibliophiles aux "Ronsardistes": collectionner Ronsard au XIX siècle, in J. Balsamo, N. Ducimetière et M. Jeanneret (dir.), Poètes, princes et collectionneurs. Mélanges offerts à M. Jean Paul Barbier-Mueller, Genève, Droz, 2011, pp. 457-472. 
sard, les neuf suivantes, posthumes, virent le jour avec une fréquence inaccoutumée qui est le signe d'une ferveur continue auprès du public. Si le jugement de certains prosateurs du XVII ${ }^{e}$ siècle, tel La Bruyère - dédaignant la "bassesse" de Rabelais et Ronsard mais appréciant la "naïveté" de Marot -, ont pu discréditer la poésie de la Renaissance aux yeux de leurs contemporains, il n'empêche que celle-ci avait rejoint très tôt les rayons des bibliothèques des Grands, des collèges et même des séminaires, et que le volume des Euvres de Ronsard figurait utilement et sans complexe aux côtés des autres auteurs classiques, Pindare et Homère, Horace et Virgile.

FRANÇOIS ROUGET

Queen's University Kingston, Ontario 


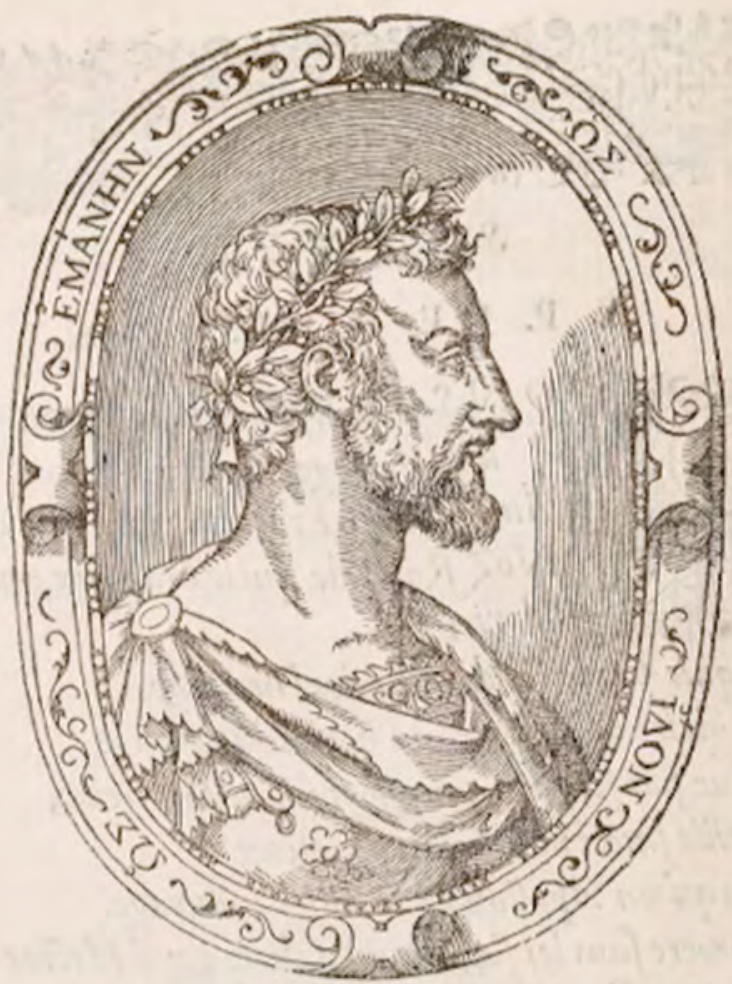

Tel fut Ronfard, autheur de cét ounrage, Tel fut fon ceil, fa bouche er fon vifage, Portrait au vif de deux crayons diners: Icyle Corps, o lEspriten fes vers.

Figure 1. Pierre de Ronsard, Euvres, Paris, G. Buon, 1584; portrait de Ronsard [ $f^{\circ}$ prélim. $6 v^{\circ}$ ]. Coll. particulière. 


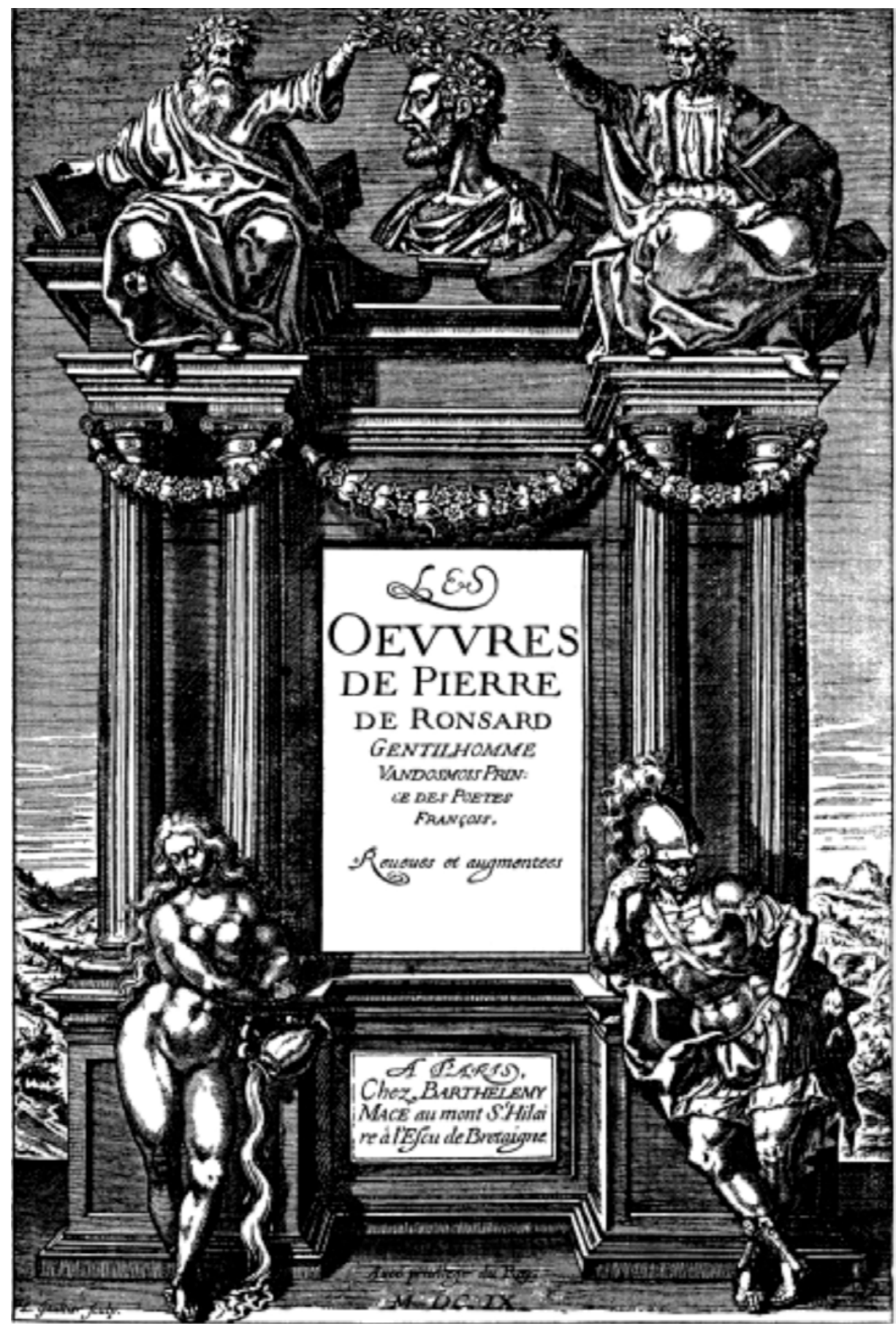

Figure 2. Pierre De Ronsard, Euvres, Paris, N. Buon, 1609; page de titre. Coll. particulière. 


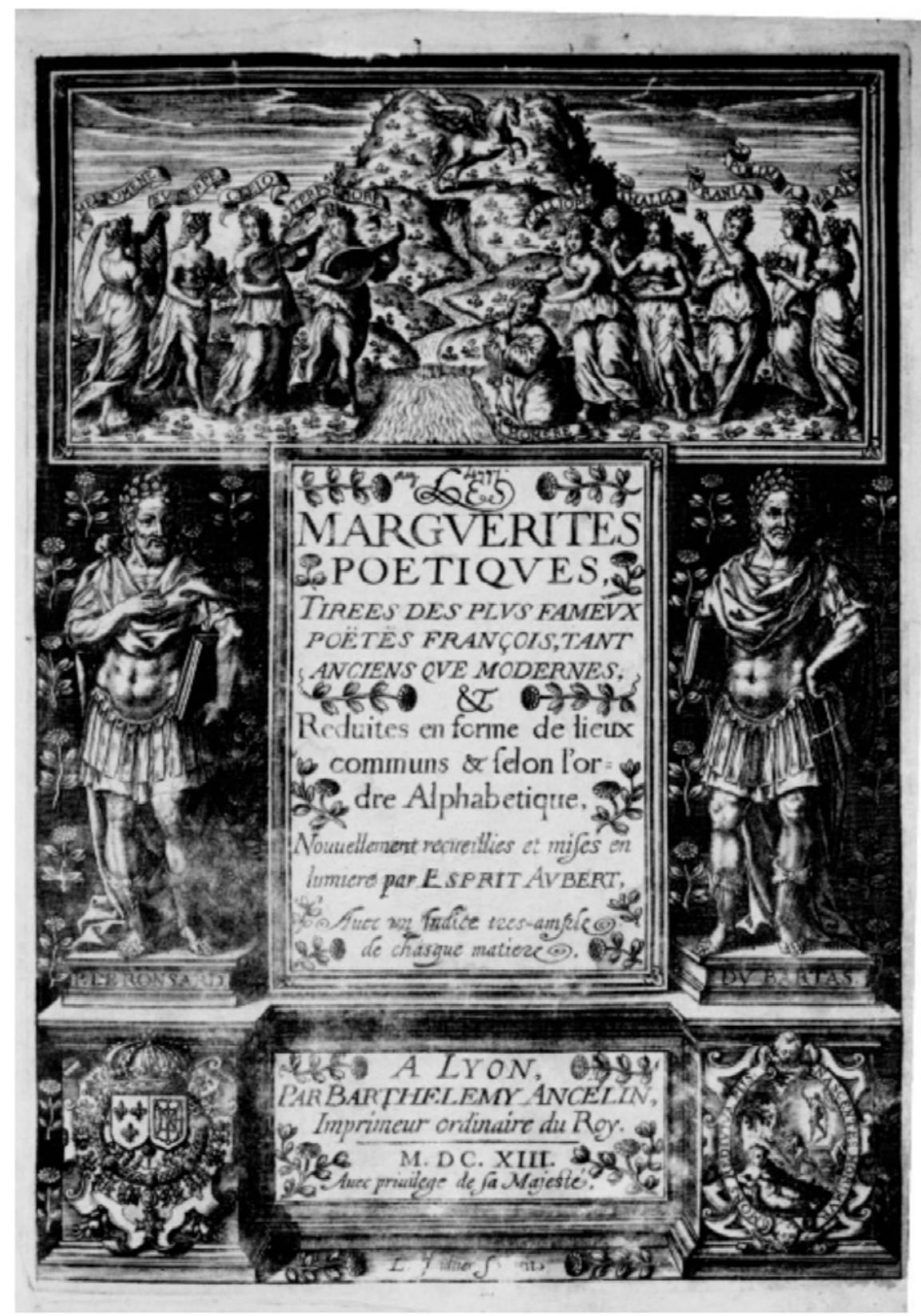

Figure 3. Marguerites poetiques, Lyon, B. Ancelin, 1613; page de titre. Coll. particulière. 


\section{Bibliographie}

I. Éditions anciennes (selon l'ordre chronologique)

Ronsard, P. De, Avantentrée du Roi Treschrestien à Paris, Paris, G. Corrozet, 1549, in $-4^{\circ}$.

Ronsard, P. DE, L'Hymne de France, Paris, M. Vascosan, 1549 , in- $8^{\circ}$.

Ronsard, P. DE, Les Quatre premiers livres des Odes [...]. Ensemble son Bocage, Paris, G. Cavellat, 1550 , in $-8^{\circ}$.

Ronsard, P. DE, Les Amours [...]. Ensemble le Cinquiesme livre des Odes, Paris, Veuve M. de La Porte, 1552 , in $-8^{\circ}$.

RONSARD, P. DE, Les Amours [...] nouvellement augmentées par lui, et commentées par Marc Antoine de Muret. Plus quelques Odes de l'Auteur, non encor imprimées, Paris, Veuve M. de La Porte, 1553, in- $8^{\circ}$.

Ronsard, P. DE, Hymne de Bacus [...]. Avec la version latine de Jean Dorat, Paris, A. Wechel, 1555 , in $-4^{\circ}$.

ThOOR, FR. DE, P. Ronsardi ad pacem exhortatio latinis versibus de Gallicis expressa a Francisco Thorio Bellione, Paris, A. Wechel, 1558.

Ronsard, P. DE, Les Euvres [...], Paris, G. Buon, 1560, in-16.

Ronsard, P. DE, Discours des miseres de ce temps. A la Royne mere du Roy, Paris, G. Buon, 1562 , in $-4^{\circ}$.

Ronsard, P. De, Continuation du Discours des miseres de ce temps. A la Royne, Paris, G. Buon, 1562, in- $4^{\circ}$.

Ronsard, P. DE, Remonstrance au peuple de France, Paris, G. Buon, 1563, in-4.

Ronsard, P. DE, Responce [...] aux injures et calomnies de je ne sçay quels Predicans, et Ministres de Geneve [...], Paris, G. Buon, 1563, in-4.

Ronsard, P. DE, Les Euvres [...] redigées en six tomes, Paris, G. Buon, 1567, in- $4^{\circ}$.

Ronsard, P. DE, Les Euvres [... r redigées en six tomes, Paris, G. Buon, 1571, in-16.

Ronsard, P. DE, Les Quatre premiers livres de La Franciade [... ], Paris, G. Buon, 1572, in $-4^{\circ}$.

Ronsard, P. DE, Les Euvres [...] redigées en six tomes, Paris, G. Buon, 1572-1573, in-16.

Ronsard, P. DE, Les Euvres [...] redigées en sept tomes, Paris, G. Buon, 1578, in-16.

Ronsard, P. DE, Les Euvres [...] reveues, corrigées et augmentées par l'Autheur, Paris, G. Buon, 1584, in-folio.

Ronsard, P. DE, Les Derniers vers [...], Paris, G. Buon, 1586, in-4 .

RONSARD, P. DE, Les CEuvres [...] corrigées et augmentées par l'autheur peu avant son trespas. Redigées en dix tomes, Paris, G. Buon, 1587, in-12.

Ronsard, P. DE, Les Euvres [...]. Augmentées de plusieurs Poësies de l'Auteur, qui n'estoyent en la precedente edition, redigées en cinq tomes, Lyon, Th. Soubron, 1592 , in-12.

RONSARD, P. DE, Les Euvres [...] reveues, corrigées et augmentées par l'autheur peu avant son trespas, et encores depuis augmentées de plusieurs Commentaires. Redigées en dix tomes, Paris, G. Buon, 1597, in-12.

RONSARD, P. DE, Les CEuvres [...] reveues et corrigées par l'Autheur peu avant son decès, augmentées en ceste edition de plusieurs pieces non encores veuës. Avecques plusieurs Commentaires sur les Amours, les Odes et les Hymnes. Redigées en X tomes, Paris, N. Buon, 1604, in-12.

Ronsard, P. DE, Les Euvres [...] reveues et augmentées, Paris, N. Buon et B. Macé, 1609 , in-folio. 
Ronsard, P. DE, Les Euvres [...] reveues et augmentées, Paris, N. Buon et B. Macé, 1609-1610, 10 t. in-12.

Ronsard, P. DE, Les Euvres [...] reveues et augmentées, Paris, N. Buon et B. Macé, 1617,10 t. in-12.

Ronsard, P. DE, Les Euvres [...] reveues et augmentées, et illustrées de Commentaires et remarques, Paris, N. Buon, 1623, 2 t. in-folio.

RONSARD, P. DE, Les CEuvres [...] reveues et augmentées, et illustrées de Commentaires et remarques, Paris, M. Henault, S. Thibout et R. Baraigne, 1629-1630, 10 t. in-12.

II. Éditions modernes (selon l'ordre chronologique)

Binet, CL., Discours de la vie de P. de Ronsard, Paris, G. Buon, 1586; éd. P. Laumonier, Paris, Hachette, 1910.

Euvres complètes de Pierre de Ronsard, éd. critique de P. Laumonier, R. Lebègue et I. Silver, Paris, STFM, 1914-1974, 20 t. (réimpression complétée par un index et des tables par J. Céard et alii, 2015).

Euvres complètes de Ronsard, éd. I. Silver, Chicago, The University of Chicago Press, et Paris, Didier, 1966-1970, 8 t.

Du Perron, J. Davy, Oraison funebre sur la mort de Monsieur de Ronsard (1586), éd. crit. de M. Simonin, Genève, Droz, 1985.

Commentaires au Premier livre des Amours de Ronsard, publiés par J. Chomarat, M.M. Fragonard et G. Mathieu-Castellani, Genève, Droz, 1985.

Le Commentaire au Second livre des Amours de Ronsard, par R. Belleau, éd. M.M. Fontaine et F. Lecercle, Genève, Droz, 1986.

Euvres complètes de P. de Ronsard, éd. crit. J. Céard, D. Ménager, M. Simonin, Paris, Gallimard, «Bibliothèque de la Pléiade», 1993-1994, 2 t.

Les Amours commentées (1553) de P. de Ronsard, éd. Chr. de Buzon et P. Martin, Paris, Didier, «Classiques», 1999.

III. Études par ordre alphabétique

Chatelain, J.-M., L'intervention de l'image et ses rapports avec le texte à la Renaissance, in La Naissance du livre moderne. Mise en page et mise en texte du livre français (XIV'-XVII siècles), éd. avec la collaboration de J.-M. Chatelain, I. Diu, A. Le Dividich et L. Pinon, Paris, Editions du Cercle de la Librairie, 2000, pp. 234-269.

Chartier, R. (dir.), Pratiques de la lecture, Paris-Marseille, Rivages, 1985.

Clement, M., Les poètes et leurs libraires au prisme du privilège d'auteur au XVI siècle: la proto-propriété littéraire, in Les Poètes français de la Renaissance et leurs "libraires", éd. D. Bjaï et F. Rouget, Genève, Droz, 2015, pp. 15-54.

Cordellier, D., Dubreuil, peintre de la "Franciade" de Ronsard au Château-Neuf de Saint-Germain-en-Laye, in «Revue du Louvre et des Musées de France» 5/6, 1985, pp. 357-378.

Dassonville, M., Ronsard. Etude bistorique et littéraire, t. I: Les enfances Ronsard (1536-1545); t. II: A la conquête de la Toison d'Or (1545-1550); t. III: Prince des Poètes ou Poète des Princes (1550-1556); t. IV: Grandeurs et servitudes (1556-1565); t. V: Un brasier sous la cendre (1565-1575), Genève, Droz, 1968-1990.

Faisant, Cl., Mort et résurrection de la Pléiade, Paris, H. Champion, 1998.

FragonarD, M.-M., Ronsard en poète: portrait d'auteur, produit du texte, in Figures du poète. Pierre de Ronsard, Actes du colloque de l'ENS de Fontenay/Saint-Cloud et 
de l'Univ. de Paris X-Nanterre, avril 1999, éd. M.-D. Legrand et J.-M. Maulpoix, Presses de l'Univ. de Paris X, «Littérales», 2000, pp. 15-41.

GabILLOT, C., Les portraits de Ronsard, in «Gazette des Beaux-Arts» XXXVII, 1907, pp. 487-501.

Huchon, M., La Fleur de poésie française dans la "Rhetorique" de Fouquelin: une autobiographie de Ronsard, in Le Poète et son cuvre. De la composition à la publication, études réunies par J.-E. Girot, Genève, Droz, 2004, pp. 215-234.

LaCHÈvre, F., Bibliographie des recueils collectifs de poésies au XVI siècle (du Jardin de Plaisance, 1502, aux Recueils de Toussaint du Bray, 1609), Paris, Champion, 1922.

Lachèvre, F., Recueils collectifs de poésies libres et satiriques publiés depuis 1600 jusqu'à la mort de Théophile (1626), Paris, Champion, 1914.

Figures du poète. Pierre de Ronsard, sous la direction de M.-D. LEgrand, M.-D. et J.-M. Maulpoix, Actes du colloque de l'ENS de Fontenay / Saint-Cloud et de l'Université de Paris X-Nanterre (avril 1999), Presses de l'Université de Paris X, «Littérales», 2000.

MaIRA, D., Typosine, la dixième muse. Formes éditoriales des canzonieri français (15441560), Genève, Droz, 2007.

MaIRA, D., Des bibliophiles aux "Ronsardistes": collectionner Ronsard au XIXe siècle, sous la direction de J. Balsamo, N. Ducimetière et M. Jeanneret, Poètes, princes et collectionneurs. Mélanges offerts à M. Jean Paul Barbier-Mueller, Genève, Droz, 2011, pp. 457-472.

Rouget, F., Ronsard et le Livre. Étude de critique génétique et d'bistoire littéraire, 2 t., Genève, Droz, 2010 et 2012.

Rouget, F., Ronsard et le grand bomme, in «Travaux de Littérature» XVIII, 2005, pp. 87-103.

Simonin, M., Ronsard et la poétique des "CEuvres", in Ronsard en son IV centenaire: I. Ronsard hier et aujourd'bui, éd. Y. Bellenger, J. Céard, D. Ménager et M. Simonin, Genève, Droz, 1988, pp. 47-59 (repris dans L'Encre et la lumière. Quarante-sept articles (1976-2000), Genève, Droz, 2004, pp. 237-251).

Simonin, M., Pierre de Ronsard, Paris, Fayard, 1990.

SмIтH, M., Ronsard et ses critiques contemporains, in Ronsard en son IVe centenaire, Actes du colloque international de Tours, éd. Y. Bellenger, J. Céard, D. Ménager et M. Simonin, Genève, Droz, 1988, t. I, pp. 83-90 (repris dans «Renaissance Studies, Articles 1966-1994», Genève, Droz, 1999, pp. 219-226).

Thibault, G. et Perceau, L., Bibliographie des poésies de P. de Ronsard mises en musique au XVI siècle, Paris, Droz, 1941. 